\title{
Vulvar Adenocarcinoma, Intestinal-Type
}

National Cancer Institute

\section{Source}

National Cancer Institute. Vulvar Adenocarcinoma, Intestinal-Type. NCI Thesaurus. Code C128166.

A rare adenocarcinoma of intestinal type that arises from the vulva. The morphological features are those of a mucinous adenocarcinoma. 